\section{Cahiers de Narratologie}

Analyse et théorie narratives

12 | 2005

Récit et éthique

\title{
Nouvelles intercalées et éthique. Autour des logiques narratives dans Eusebio (1787)
}

\section{Marc Marti}

\section{(2) OpenEdition}

1 Journals

Édition électronique

URL : http://journals.openedition.org/narratologie/24

DOI : 10.4000/narratologie.24

ISSN : 1765-307X

Éditeur

LIRCES

\section{Référence électronique}

Marc Marti, « Nouvelles intercalées et éthique. Autour des logiques narratives dans Eusebio (1787)», Cahiers de Narratologie [En ligne], 12 | 2005, mis en ligne le 08 juillet 2010, consulté le 15 novembre 2019. URL : http://journals.openedition.org/narratologie/24 ; DOI : 10.4000/narratologie.24

Ce document a été généré automatiquement le 15 novembre 2019.

\section{(c) (i) (9)}

Cahiers de Narratologie - Analyse et théorie narratives est mis à disposition selon les termes de la licence Creative Commons Attribution - Pas d'Utilisation Commerciale - Pas de Modification 4.0 International. 
Nouvelles intercalées et éthique. Autour des logiques narratives dans Eusebio (1787)

\author{
Marc Marti
}

1 S'interroger sur les relations entre le récit et l'éthique ouvre la voie à plusieurs approches. Nous avons ici choisi une double perspective. D'une part, celle d'une sémiologie des valeurs telle que la définit Vincent Jouve, c'est-à-dire répondant à la question "par quels procédés le texte rend-t-il perceptibles les valeurs dont il se réclame? $»^{1}$. D'autre part, celle d'une sociocritique telle que la définit Edmond Cros dans son dernier ouvrage :

En fait la sociocritique ne s'intéresse pas à ce que le texte signifie, mais à ce qu'il transcrit, c'est-à-dire à ses modalités d'incorporation de l'Histoire, non pas d'ailleurs au niveau des contenus, mais au niveau des formes ${ }^{2}$.

Dans ce cadre, nous proposons d'analyser la logique ou combinatoire narrative d'un roman espagnol du 18e siècle, Eusebio (1787) de Pedro Montengón, à travers les relations entre le récit principal et les nouvelles intercalées.

3 Ce roman est certainement un des plus étudiés de la littérature espagnole du 18e siècle mais jusqu'à présent, c'est surtout sa thématique qui a suscité l'intérêt de la critique. Souvent considéré comme une œuvre exemplaire, il est bien plus analysé pour sa valeur comme document philosophique que comme œuvre de fiction ${ }^{3}$. Pourtant, les modèles narratifs que convoque cette œuvre sont multiples et leur analyse permettrait d'en nuancer la portée exemplaire.

Dans un travail précédent ${ }^{4}$, nous avions étudié les fonctions des nombreuses nouvelles intercalées présentes dans le roman. Nous désirerions ici prolonger cette analyse en nous interrogeant sur le discours éthique qui motive la logique narrative de ces récits secondaires.

D'abord, il s'agira de déterminer l'objet et la méthode de travail. Définir les nouvelles intercalées n'est pas chose aisée, et la tâche est peut être même impossible. Cependant, un éclaircissement sera profitable. Pour ce qui est de la méthode, nous nous inspirerons 
directement des travaux de Jean Michel Adam sur la structure du récit ${ }^{5}$. Le modèle logique qu'il propose nous semble le plus adapté pour analyser les combinatoires narratives de Eusebio.

6 Dans un second temps, il faudra décomposer les nouvelles intercalées, les comparer entre elles, puis les apprécier en fonction du récit principal.

7 Le résultat de cette analyse devrait nous amener à formuler quelques hypothèses sur la façon dont l'éthique sous-tend la structure du récit dans son ensemble et la façon dont celui-ci retranscrit à son tour la doxa.

\section{Le corpus et la méthode}

\subsection{Nouvelle et nouvelles intercalées : quelques principes généraux}

Il ne s'agit pas ici de revenir sur les difficultés que pose la définition de la nouvelle, problème abordé dans un précédent travail ${ }^{6}$. Nous retiendrons que la nouvelle est un genre narratif à part entière tout en reconnaissant que la critique lui attribue une définition particulièrement fluctuante ${ }^{7}$. Cette identité générique mal assurée tient en grande partie à l'histoire d'un genre non-aristotélicien : le terme n'a pas recouvert la même réalité dans la littérature classique et dans la littérature contemporaine. Le même problème se pose d'ailleurs pour le roman.

9 Ce préalable posé, il n'en demeure pas moins que le roman de l'âge classique se caractérise souvent par l'inclusion de nouvelles intercalées, et assumées comme telles par les auteurs 8 . Dans la littérature hispanique, celles-ci ont finalement été très peu analysées sous l'angle du jeu narratif. Seul Jean-Louis Brau, dans son travail de thèse, s'est intéressé au problème de façon systématique à partir du Guzmán de Alfarache et de la première partie du Don Quichotte ${ }^{9}$. Les critères qui lui ont permis de constituer un corpus pour chacun des deux romans ébauchent une théorie du genre intercalé qui nous servira de base pour la suite. Dans le texte de Mateo Alemán quatre nouvelles sont retenues car elles sont racontées par un narrateur externe et différent de celui de la narration principale. Une cinquième nouvelle $d u$ corpus ne suit pas ce critère (La veuve vengeresse), puisque narrée par Guzmán lui-même. C'est sa thématique qui en fait une nouvelle intercalée. Par ailleurs, les nouvelles intercalées du Guzmán peuvent parfaitement être délimitées à partir de la voix narrative. Chaque fois, elles sont narrées par un personnage qui appartient à la diégèse (récit principal), mais ce narrateur est complètement extérieur à l'action de la nouvelle ${ }^{10}$.

Le cas du Quichotte est beaucoup plus complexe. «Le fait même que les personnages des nouvelles coexistent avec ceux de la narration principale rend difficile la détermination des limites respectives des deux types de récits. Où s'arrête le récit des nouvelles et où commence celui du roman? C'est souvent impossible à dire $»^{11}$. Après cette constatation, Jean-Louis Brau propose de retenir sept nouvelles intercalées, à partir de critères qui ne font plus seulement intervenir l'interpolation car, dans ce cas, seul le récit $E l$ curioso impertinente serait valable. En effet, les autres nouvelles sont narrées par des personnages qui sont soit les acteurs, soit les témoins de l'histoire rapportée.Une thématique amoureuse, différente de celle la trame principale, sert alors de démarcation, comme dans les nouvelles de Marcela y Grisóstomo ou Dorotea y don Fernando. Mais dans ce dernier cas par exemple, la protagoniste féminine joue aussi un rôle dans la diégèse, en se travestissant en princesse Micomicona. Par ailleurs, cette 
nouvelle, dont une partie de l'action est aussi imbriquée dans celle de Cardenio y Luscinda, est racontée par trois narrateurs différents. Le début est assuré par Dorotea, alors que le dénouement est rapporté par le narrateur principal du roman, quelques chapitres plus loin. À ces deux principales sources d'information, il faut ajouter une partie du récit de Cardenio, qui complète les informations lacunaires sur le mariage entre Luscinda et Don Fernando. Le cas du Quichotte, bien avant l'œuvre de Montengón, pose donc déjà le problème des limites de la nouvelle intercalée et de son absorption « incomplète » par la trame romanesque.

\subsection{La nouvelle intercalée, un matériau narratif hétérogène?}

11 En effet, pour que la nouvelle intercalée existe, il faut que l'absorption par la trame romanesque soit inachevée. En d'autres termes, les marques d'une altérité générique doivent être visibles, car dans le cas contraire, la nouvelle intercalée basculerait totalement dans la trame romanesque. C'est sur ces marques d'altérité que devrait reposer une définition du genre intercalé.

12 Le premier trait pourrait être l'architecture narrative générale du roman. La narratologie propose les termes de récit premier et récit second ou métarécit. Selon Gérard Genette " est récit second tout récit pris en charge par un agent de narration (ou plus généralement de représentation) intérieur au récit premier ${ }^{12} »$.Pour que le lecteur perçoive la nouvelle intercalée comme telle, le changement de narrateur semble obligatoire.

Cependant, cette règle n'est pas une constante. Dans le roman de Montengón, deux des six nouvelles intercalées sont entièrement prises en charge par un personnage du récit premier, les quatre autres fonctionnent sur un modèle mixte qui rappelle celui du Quichotte. Généralement un personnage commence à raconter une histoire dont le dénouement aura lieu dans le récit premier et sera raconté par le narrateur principal ${ }^{13}$.

Du point de vue esthétique et formel, il semblerait que dans la littérature hispanique on ait assisté à une évolution intéressante. On serait passé de la collection de nouvelles encadrées par une trame narrative servant presque uniquement à mettre en scène la narration à une trame où le cadre prend progressivement le dessus sur les nouvelles ${ }^{14}$. Cette coexistence de deux schémas narratifs imbriqués est considéré par Edmond Cros comme un processus de gestation du roman en tant que genre autonome. Dans une certaine mesure, ce dernier serait le résultat d'une hybridation du recueil de nouvelles et de l'Histoire ${ }^{15}$. La fluctuation terminologique dont il a été l'objet en castillan témoignerait de ce croisement. Le terme qui s'est finalement imposé désigne à la fois le roman (novela) et la nouvelle (novela corta). Ce constat nous amène à nous interroger sur les relations complexes que suppose la présence de nouvelles dans le roman, problème que nous aborderons sous l'angle des logiques narratives et de l'éthique.

\subsection{La logique narrative, une logique éthique?}

En se fondant sur les conclusions des travaux de Jean-Michel Adam sur le texte narratif, nous considèrerons que toute séquence narrative est composée de sept macropropositions ${ }^{16}$. Cette division permet de considérer le récit à partir d'un schéma 
quinaire, un modèle proposé par Paul Larivaille (1974), qu'Adam reprend dans ses travaux ( $\mathrm{Pn}=$ Macro-proposition narrative) et expose de la façon suivante :

- Pn1: Situation initiale (orientation)

- Pn2: Complication, Déclencheur 1

- Pn3: Actions ou évaluation

- Pn4: Résolution, déclencheur 2

- Pn5: Situation finale

Les macro-propositions peuvent à leur tour être décomposées en un nombre $n$ de propositions, mais nous n'entrerons pas dans cette ultime division qui ne relève plus seulement de l'analyse logique du récit mais aussi de la linguistique. Cette segmentation, basée sur la logique des événements, ne rend pas compte de la totalité du texte narratif, qui est traversé aussi par des éléments évaluatifs. Pour analyser cet ensemble (la plupart du temps hétérogène) qu'est le texte narratif, J. M. Adam propose de considérer que l'architecture de la séquence narrative de base (modèle quinaire) comporte deux macro-propositions supplémentaires, qui auront pour rôle d'encadrer le récit et de favoriser son interprétation dans le sens désiré par son énonciateur. Elles ne sont pas des éléments du récit à proprement parler, mais elles font partie du texte narratif. Il conviendra donc de "distinguer le commencement d'un récit (qu'on appelle l'Orientation Pn1) de l'ouverture du tour de parole narratif ("entrée préface" ou Résumé), comme on va distinguer [...] la fin du récit (Situation finale-Pn5) de la clôture du tour de parole (la "Chute" ou "Coda") ${ }^{17}$ ». Aux cinq macro-propositions narratives constitutives du récit viennent s'ajouter les deux suivantes, la première au début, la seconde à la fin:

Pn0: Résumé et/ou entrée préface

$\operatorname{Pn} \Omega$ : Chute ou morale.

19 Adam précise «qu'il y a une nette différence entre les Chutes, dépourvues d'évaluation et uniquement destinées à marquer la fin de la prise de parole narrative et celles qui tirent des conclusions pour le présent et ramènent donc à la situation d'énonciation et aux tours de parole éventuels. La Morale qui vient clore le récit et le tour de parole apparaît en dehors du corps du récit ${ }^{18}{ }^{\text {». }}$.

Cette méthode d'analyse répond parfaitement à notre intention. En effet, s'il semble évident que les "entrées-préfaces" et les "morales » sont des lieux investis par le discours éthique et la plupart du temps analysés comme tels ${ }^{19}$, il n'en demeure pas moins que d'autres moments de la séquence peuvent aussi être porteurs de valeurs. Si l'on examine les 5 autres macro-propositions, elles peuvent aussi être révélatrices.

D'abord, la situation initiale (Pn1) est toujours une situation "d'équilibre». Son examen pourrait révéler ce que le récit propose comme étant «normal » ou stable. La complication ( $\mathrm{Pn} 2)$ est sans nul doute encore plus révélatrice. Elle provoque la rupture de la stabilité, c'est l'élément perturbateur, « l'anormalité » nécessaire pour qu'il y ait récit. Elle se concrétisera par une " agression " ou une "transgression " si l'on reprend les termes de Vladimir Propp ${ }^{20}$. L'action (Pn3) est sans doute, dans le déroulement logique de la séquence narrative, la macro proposition la plus neutre. Découlant de la complication, sa valeur dans l'analyse de l'éthique peut sembler moindre. Cependant, les moyens mis en œuvre dans l'action peuvent être l'objet d'une évaluation. La résolution (Pn4) pourra révéler le discours éthique, en suggérant par quels moyens il est licite de rétablir l'équilibre et de contrecarrer la complication. On rappellera cependant «qu'elle peut ne manifester aucune résolution au problème posé [...]; ce 
second déclencheur permet à la séquence de s'achever comme le premier (Pn2) assurait, de son côté, le démarrage de la séquence ${ }^{21} »$. La situation finale, (Pn5) est à rapprocher de la situation initiale. Elle offre habituellement un nouvel équilibre qui peut correspondre soit à une amélioration, soit à une dégradation.

Cette vision doit être néanmoins nuancée. Elle laisserait en effet penser que le poids de la logique narrative enferme le récit dans une structure "conservatrice», qui aurait horreur, non du vide, mais du déséquilibre. En fait, tout dépendra à quel niveau on se situe. Du point de vue de l'action, il semble évident que le récit - du moins dans ses avatars de l'époque classique- tend toujours vers la complétude. Du point de vue de l'éthique, il pourra en être tout autrement. La situation finale peut par exemple être en contradiction avec la morale en vigueur ou celle de la situation initiale. L'examen des nouvelles intercalées dans le roman Eusebio permettra de préciser ces différences.

\section{Analyse des nouvelles intercalées dans Eusebio}

\subsection{Le corpus et le mode narratif}

Dans un précédent travail sur le roman de Montengón qui portait sur la théorie des genres intercalés, nous avions défini six nouvelles, dont nous reprenons l'argument en annexe de ce travail ${ }^{22}$ :

- I. pp. 129-149 et pp. 414-418 : La vie de John Bridge.

- II. pp. 235-244 : Histoire de la passion amoureuse excessive d'Omfis.

- III. pp. 247-261 : Histoire d'Isidoro, version contraire de la précédente.

- IV. pp. 339-356: Histoire de Bridway, le noble devenu cordonnier.

- V. pp. 548-560 : Histoire d'Adelaïde, jeune fille séduite par un libertin.

- VI. pp. 675-85 : Histoire de Lord Towsend, un noble exilé.

Les six nouvelles se trouvent dans les deux premiers tiers de l'œuvre. La dernière partie du roman n'en contient aucune. Il faudra nous interroger sur cette distribution en analysant les fonctions remplies par ces récits. Avant de considérer leur portée éthique à partir de l'analyse des séquences narratives, il convient de préciser leur mode d'interpolation.

Nous avions retenu, comme critère de définition de la nouvelle intercalée, la nécessité d'un narrateur au second degré. Toutes les histoires du corpus sont bien racontées par des personnages fictifs. Cependant, elles ne sont pas toutes interpolées de la même façon. Une partie de la narration des nouvelles I, IV, V, VI, est assurée par le narrateur principal, car il fait intervenir Eusebio et Hardyl, qui en deviennent alors momentanément les personnages et non plus les simples auditeurs. Le cas de la I est très particulier, puisque J. Bridge reçoit de l'argent de Hardyl, qui lui permettra de revenir en Angleterre, mais il y aura des rebondissements, qui seront racontés à nouveau par le personnage presque trois cents pages plus loin. Dans ce cas, le narrateur principal prend en charge le milieu de la nouvelle alors que pour les trois autres, c'est le dénouement qui s'intègre ainsi à la trame du récit principal. En IV, Bridway, qui a tout perdu, se console de son triste sort par le spectacle de vertu que lui offrent Eusebio et Hardyl. En V, les deux héros permettent à Adelaïde d'échapper à l'hôpital de Bicêtre et de rentrer chez ses parents. En VI, lord Towsend retrouve l'aisance matérielle grâce à la générosité d'Eusebio. Finalement, seules les nouvelles II et III sont entièrement racontées par le même narrateur (Hardyl) mais celui-ci n'en est pas le protagoniste. 
Celles-ci ont un statut particulier, car elles ne sont pas, en apparence des récits " autobiographiques », contrairement aux quatre nouvelles (I, IV, V, VI) ${ }^{23}$. Par ailleurs, dans ce dernier groupe, les narrateurs jouent ensuite un rôle dans la trame principale, intégrant ainsi étroitement la nouvelle au déroulement général du roman. John Bridge, héros et narrateur de la nouvelle I est le cas le plus représentatif. Aidé par Hardyl à Philadelphie, on le retrouve presque trois cent pages plus loin à Londres. Il portera secours à son tour à Hardyl et Eusebio et les accompagnera pendant une partie de leur séjour londonien. Bridway hébergera les deux protagonistes dans sa pauvre maison au début de l'épisode anglais et, après avoir retrouvé sa fortune, Eusebio le remerciera généreusement. Adelaïde, (nouvelle V), et lord Towsend (nouvelle VII) seront, eux aussi, les bénéficiaires de la bonté d'Eusebio.

Finalement, du point de vue de la narration, seules les nouvelles II et III semblent moins intégrées à la trame. Elles sont prises en charge par Hardyl, qui en fait des récits exemplaires, exposés sous forme de diptyque : d'un côté le mauvais exemple d'Omfis, de l'autre le bon exemple d'Isidoro. Ces personnages sont ensuite totalement absents de la trame principale. Les similitudes devront être recherchées à un autre niveau.

\subsection{Analyse comparative des macro-propositions}

Nous renvoyons à l'annexe pour l'argument de chaque nouvelle, afin de faciliter la compréhension de l'analyse comparative des séquences narratives qui les composent.

\subsubsection{Les situations initiales, les complications et l'action}

Les situations initiales de toutes les nouvelles mettent en scène des personnages fortunés ou nobles. Isidoro (III), qui malgré la noblesse et la richesse de sa famille est condamné à la médiocrité par sa situation de second, n'en demeure pas moins noble.

Les complications sont assez identiques. On remarque en effet qu'elles se traduisent toutes par l'arrivée d'un agresseur, pour reprendre les termes du structuralisme. Cet agresseur est extérieur dans les nouvelles III, IV, V, VI, alors que dans les nouvelles I et II, c'est le personnage qui est son propre agresseur en laissant libre cours à ses pulsions -la vengeance et la luxure-, transgressant la doxa représentée par Eusebio et Hardyl. Dans tous les récits, les protagonistes principaux sont atteints par une déchéance matérielle puis morale. Les actions qui suivent la complication ne font que confirmer la dégradation. John Bridge perd sa situation de riche héritier en s'exilant et tombe de Charybde en Scylla, Omfis se ruine à cause de son aveuglement amoureux, Bridway voit ses possessions confisquées, Adelaïde perd son honneur et à la suite son rang, Lord Towsend perd sa haute charge de gouverneur. La nouvelle III fait cependant exception à la règle, car le changement de situation est volontaire: Isidoro choisit de se marier avec une paysanne de son plein gré, tout comme il décide de devenir agriculteur. Le renoncement à son statut de noble est largement compensé par un bénéfice moral, celui d'une vie heureuse et vertueuse à la campagne.

\subsubsection{Résolutions et situations finales}

Dans le cas des résolutions et des situations finales, si on excepte les nouvelles II et III, qui sont intégralement extra-diégétiques, les autres font toutes intervenir Eusebio et Hardyl. Bridge retourne en Angleterre grâce aux deux héros et son crime est 
heureusement effacé par la justice. Bridway ne s'apitoie plus sur son sort face à l'exemple d'humilité offert par Eusebio et Hardyl. Adelaïde est sauvée de l'enfermement et le retour chez ses parents est négocié par Hardyl, qui passera sous silence son activité passée de prostituée. Towsend sera secouru par les dons d'argent d'Eusebio. Dans l'ensemble des nouvelles, la résolution permet de retrouver la sécurité matérielle. Cependant, celle-ci reste marquée, pour un certain nombre de personnages, par le compromis et la passivité. Les protagonistes bénéficient de l'aide extérieure et ne sont pas vraiment les acteurs de leur salut.

Bridge revient en Angleterre, mais il doit sa prospérité retrouvée à l'oubli providentiel de ses deux crimes. Le premier, qui faisait suite à une rixe a été " pardonné » d'une certaine façon, mais le second, parfaitement prémédité, reste impuni. Pour Adelaïde, le dénouement est nettement marqué par un arrangement. Ses parents acceptent son retour, mais Hardyl se garde bien de les informer du temps qu'elle a dû passer à se prostituer.

Dans le même ordre d'idée, tous les "agresseurs», bénéficient d'impunité dans ces récits intercalés : Kirke et Jeffreys peuvent jouir du bénéfice de leurs crimes, Lorvál [sic] échappe à la justice et il fait même une réapparition furtive dans l'auberge d'Eusebio, Lord W... pourra tranquillement persécuter Towsend sans être inquiété par la suite.

Ces nouvelles sembleraient proposer un modèle narratif où une partie des personnages échappent à la finitude du récit, dans le sens où tout crime ne serait pas nécessairement puni. On s'orienterait alors vers un modèle narratif plus ouvert, un modèle où le récit n'aurait pas besoin de se refermer entièrement sur une situation finale où toutes les «boucles » narratives seraient bouclées. Ce modèle utiliserait au contraire l'incomplétude, ce qui laisserait penser qu'on abandonne au lecteur le soin de condamner ou non les coupables.

Cette conclusion est cependant provisoire. Les nouvelles intercalées ne sont pas des pièces autonomes. Elles s'insèrent dans un ensemble plus vaste et il semble bien que le modèle narratif ouvert qu'elles actualisent entre en conflit avec celui des épisodes principaux du roman.

\subsection{La logique des nouvelles est-elle celle du roman?}

En effet, lorsqu'on s'intéresse aux aventures d'Eusebio, on ne peut que constater que celui-ci doit toujours son salut à lui-même et à son éducation. Il n'y a pas d'intervention providentielle ni extérieure. Par ailleurs, un des éléments les plus frappants est que tous les personnages qui s'en prennent au héros ou à ses proches sont systématiquement châtiés ${ }^{24}$. Le déroulement du roman en offre de nombreux exemples.

Le chirurgien qui prétend hériter de la maison de Hardyl sera démasqué et il mourra dans l'abordage du navire qui l'emmenait en Europe ${ }^{25}$. Orme, qui tente d'enlever et de violer Leocadia ${ }^{26}$, future femme d'Eusebio, mourra sur l'échafaud en Angleterre, en compagnie de Felipe Blund, qui avait fait injustement accuser de vol et emprisonner les deux héros lors de leur séjour à Londres ${ }^{27}$. Cette exécution conjointe est le fruit du "hasard», mais les deux délinquants avaient auparavant été pardonnés par Eusebio. Lors de l'épisode parisien, Lord Som..., qui menait une vie dissolue et qui avait essayé de 
tenter Eusebio en organisant un repas avec des prostituées sera frappé par la maladie et mourra après s'être repenti ${ }^{28}$.

Enfin, au cours du dernier épisode européen (le retour en Espagne), Don Felipe pris de passion pour Leocadia essaiera d'abuser d'elle malgré la possibilité d'un lien de parenté. Ne parvenant pas à ses fins, il fera emprisonner le couple. Le confesseur de son père lui révèle peu après qu'il est un enfant adopté et le frère de la femme qu'il convoitait. L'accès de remords le conduira à se suicider.

Ce trajet des agresseurs est en accord avec celui des victimes. En effet, toutes ces épreuves sont franchies avec succès, soit par Eusebio, soit par Hardyl, soit par Leocadia. Le résultat en est toujours une amélioration morale, sans perte du statut matériel de départ, ce qui n'est pas toujours le cas pour les personnages principaux des nouvelles intercalées. Ces deux façons de développer le récit nous amènent à nous interroger sur les raisons qui peuvent motiver un tel choix esthétique, un choix sans nul doute tributaire du propos éthique.

\section{Logique, esthétique et éthique}

\subsection{Des logiques complémentaires}

La comparaison entre les macro-propositions initiales et finales dans le récit premier et le récit second mettent donc clairement en évidence une opposition. Cette opposition pourrait d'abord être considérée comme un effet de surface. En effet, les nouvelles sont subordonnées au roman et, dans un premier temps, leur narrataire est Eusebio et non le lecteur. Elles sont toujours présentées comme exemplaires et « réelles ». N'oublions pas les préfaces que propose Hardyl à Eusebio ou bien les évaluations qui servent de conclusion à ces épisodes. Juste avant le récit de John Bridge, Hardyl avait enseigné à Eusebio la nécessité de contrôler la pulsion de vengeance. L'illustration par l'histoire d'un jeune homme victime de cette pulsion ne saurait être plus claire. Parfois, l'ordre chronologique est inverse. Eusebio résistera à une prostituée en pensant au récit d'Adélaïde qui précède sa tentation ${ }^{29}$. Cette exemplarité est ensuite démultipliée par la structure du roman. Eusebio sert de relais au lecteur, qui est invité à voir en lui une personne pourvue d'une éducation qui lui permet de surmonter tous les aléas de la vie, ce qui n'est pas le cas des personnages des nouvelles. L'effet cadre vient verrouiller l'interprétation des récits seconds dans le sens des idées développées au niveau du récit premier.

C'est ainsi qu'il convient d'interpréter les " châtiments » car ils suggèrent le triomphe absolu du modèle éducatif proposé. Par sa complétude, trame principale semble plus idéaliste que la trame secondaire, où domine le compromis. On remarquera aussi que ce compromis est loin d'être le modèle à suivre. En effet, dans tous les cas, les séquences narratives des nouvelles secondaires débouchent sur une situation finale dégradée. Certes les personnages retrouvent l'aisance matérielle, mais ils ne sortent pas grandis moralement, à la différence des héros du récit premier. L'ensemble du roman constitue une synthèse entre un modèle de vie idéal, celui d'Eusebio, et un modèle de vie dégradée, celui des personnages de nouvelles.

$41 \mathrm{Du}$ point de vue esthétique, ces nouvelles semblent par ailleurs introduire une préoccupation pour la vraisemblance, étroitement liée à l'exemplarité. La façon dont Hardyl ouvre son récit de la vie d'Isidoro est révélatrice : 
Esto me trae a la memoria el caso de un dichoso casamiento; y puedes creer que no lo tomo de Filemón y Baucis. Tales historias son demasiado lejanas para que hagan impresión en nuestros pechos. El caso es reciente, es el de un joven amigo mío, el cual también contribuyó para que yo escogiese la vida que llevo ${ }^{30}$. « reciente » et « lejano ». Mais le propos de cette vraisemblance reste l'implication du lecteur. L'histoire doit le toucher («impresión en nuestros pechos »). Joaquín Álvarez Barrientos, estime que c'est dans les récits intercalés que Montengón fait preuve de tous ses talents de romancier ${ }^{31}$. Cette remarque est à la fois celle d'un critique littéraire et d'un lecteur contemporain. Notre conception de la littérature s'accommode mal d'un modèle narratif idéaliste et moralisateur tel que celui de la trame principale, et il est évident que les nouvelles constituent, de ce point de vue, un beau contrepoint, bien plus attractif, en venant problématiser les comportement sociaux et moraux. Cependant, c'est bien l'ensemble qu'il convient de juger et il faudra estimer dans quelle mesure il renvoie à une nouvelle esthétique, indissociable d'une éthique, celle de l'Espagne des Lumières.

\subsection{Une nouvelle esthétique au service de l'éthique}

Le texte de Montengón offre un nouveau modèle de littérature. Les éléments traditionnels sont abandonnés au profit « d'exemples » ancrés dans le présent. On aura $\mathrm{du}$ mal à y retrouver des historiettes ou un quelconque fonds de récits folkloriques. Dans un travail précédent, nous avions insisté sur cette nouvelle conception du roman $^{32}$. Le modèle à éviter est incarné par les narrations avortées et extravagantes du personnage populaire Gil Altano, valet d'Eusebio. Le seul épisode à coloration folklorique ${ }^{33}$, la chasse aux fantômes («duendes») dans une maison hantée, sert de support à une démystification, qui renvoie définitivement les croyances populaires aux oubliettes de l'ignorance.

Mais on s'éloigne aussi de la conception aristotélicienne et classique de la littérature en proposant une autre définition de la vraisemblance, fondée non plus sur l'imitation de la nature particulière ou universelle mais sur l'expérience individuelle du monde ${ }^{34}$. Le choix est à la fois éthique et esthétique.

Par l'imperfection de leur dénouement, les nouvelles servent la vraisemblance romanesque et affirment, dans une certaine mesure, la valeur de l'expérience individuelle. En outre, une nette évolution est perceptible par rapport au siècle précédent. Tous les échecs conduisent à la perte non de l'honneur, mais d'une certaine aisance matérielle, sauf pour Adélaïde qui, en tant que femme, perdra les deux, mais n'en sera pas pour autant condamnée au couvent. Le roman propose au bout du compte une casuistique du bonheur et des moyens pour y parvenir, préconisant un stoïcisme modernisé, fondé sur les leçons de l'expérience vécue que représentent les nouvelles ${ }^{35}$ La dialectique entre les deux niveaux du récit, exprimée par des programmations narratives différentes met aussi à jour une préoccupation esthétique et éthique. D'une part il s'agit de proposer un texte édifiant, ce qui implique, du point de vue littéraire, un certain «affadissement » du roman tel qu'il est en train de s'imposer en Europe. D'autre part, il faut maintenir l'attention du lecteur et c'est ici qu'interviennent les épisodes intercalés, où le ton est bien moins moralisateur par le choix d'une narration autobiographique et des boucles narratives moins prévisibles. Mais cette concession constitue un danger pour le propos éducatif et la doxa. Pour cette raison, le récit 
premier vient servir de contrepoint aux nouvelles en les encadrant fortement. Cette dialectique est sous-tendue par le classique précepte d'Horace qui recommandait de joindre l'utile à l'agréable ${ }^{36}$. L'idée avait été réactivé par le néoclassicisme espagnol qui en faisait le fondement de sa conception de la littérature. Derrière cette préoccupation esthétique, on peut voir une méfiance certaine vis-à-vis de la littérature de divertissement. Elle pouvait constituer un danger par l'émancipation du lecteur, qui se retrouvait face à des œuvres d'autant plus agréables à lire qu'elles étaient, dans une certaine mesure, transgressives. Le roman de Montengón semble faire le pari d'intégrer à la littérature édifiante cette autre littérature, ce qui lui vaut la reconnaissance de la critique contemporaine comme romancier de référence pour le $18^{\mathrm{e}}$ siècle espagnol ${ }^{37}$.

\section{Pour conclure}

Bien que notre travail soit essentiellement fondé sur une recherche de l'éthique dans le roman, plusieurs autres voies restent ouvertes.

D'abord, la typologie du genre intercalé que nous avons ébauchée pour définir notre objet d'étude devra être complétée. En effet, une recherche narratologique plus approfondie sur ce point nous semble indispensable, dans la continuité des travaux de Jean-Louis Brau. L'analyse du schéma narratif des nouvelles intercalées nous a permis de mettre en relief un certain nombre d'invariants. Ceux-ci n'ont pu être interprétés qu'à la lumière de l'ensemble de l'œuvre.

Ensuite, une nouvelle conception esthétique semble émerger. L'idée que seule la "réalité » peut être exemplaire induit que les épisodes de l'oeuvre de fiction doivent rester les plus vraisemblables possible. Ce but nous semble peut être mieux concrétisé dans les nouvelles intercalées et leur incomplétude, à l'image de la vie. Cette remarque reste cependant en partie celle d'un lecteur contemporain. En effet, en y regardant de plus près, ces nouvelles doivent peut être beaucoup aussi à une littérature de genre, insérée à dessein dans ce roman d'éducation.

Dans une perspective plus large, ce travail demanderait à être prolongé dans deux directions. D'une part, il faudrait prendre en compte la pratique du récit secondaire et du récit intercalé dans le roman des Lumières. Il s'agit d'une technique narrative particulière, qui sans être nouvelle, demande à être mieux cernée afin d'en saisir les éventuelles significations ${ }^{38}$. D'autre part, la nouvelle au $18^{\text {ème }}$ siècle semble un genre oublié par l'histoire de la littérature, alors que des écrivains espagnols s'y consacraient, soit comme traducteurs (souvent adaptateurs), soit comme créateurs originaux.

\section{ANNEXES}




\section{Arguments des six nouvelles intercalées dans Eusebio}

I. pp. 129-149 et pp. 414-418. La vie de John Bridge est la première nouvelle intercalée dans le roman. Ce jeune anglais, mal éduqué, fils d'un riche commerçant, a tué un noble lors d'une dispute. Il a dû s'exiler volontairement en Amérique où il débarque ruiné, après avoir perdu tout son argent dans un naufrage. Il entre dans l'armée. Il va épouser sur un coup de tête la fille d'un tambour. Sa femme devenant insupportable, il l'entraîne en forêt pour l'assassiner. Une fois son forfait accompli, il est fait prisonnier par les indiens, avec lesquels il va vivre un bon moment. Il réussit à s'échapper et finit comme mendiant. C'est à ce moment qu'il croise la route d'Hardyl et d'Eusebio. Après l'avoir accueilli, Hardyl lui donne de l'argent pour qu'il rentre en Angleterre. On le retrouve plus tard lors du séjour européen des deux héros. Grâce à des circonstances favorables, son premier crime a été pardonné. Il a pris sa place de riche commerçant et s'est marié. Il aidera Hardyl et Eusebio injustement accusés de vol. Il ne s'est pas totalement amendé car il entretient une passion dévorante pour le jeu.

II. pp. 235-244. La seconde nouvelle met en scène l'histoire de la passion amoureuse excessive d'Omfis. Elle est racontée de façon linéaire par Hardyl. Omfis, jeune homme, riche, beau et noble aime Earina. Leur passion est contrariée par le père du garçon. Après un malentendu provoqué par Omfis, son père est tué lors d'un duel avec le père d'Earina et le mariage désiré peut avoir lieu. Les jeunes époux vont mener la grande vie et se couvrir de dettes, ce qui va provoquer des tensions dans le couple. Earina se laisse séduire par un de ses cousins, Silio. Omfis les surprend, mais doit, sous la menace, jurer de ne pas faire de mal à sa femme. À partir de ce moment, il vit en ignorant sa femme, qui continue à voir son amant. Silio meurt de façon spectaculaire dans les bras d'Earina, Omfis découvre qu'il est à nouveau trompé et tue sa femme.

III. pp. 247-261. La nouvelle trois est une sorte de contrepoint de la précédente. Isidoro est le second d'une famille noble. Il connaît un premier échec amoureux auprès d'une riche héritière qui va lui préférer un " mayorazgo ». Il se console en cultivant sa vertu par la lecture. Il tombe amoureux de Dorothée, une paysanne, mais sa famille, prévenue par un rival, s'oppose à ce mariage inégal. Il feint alors de partir en Italie et va se marier secrètement avec Dorotée. Ils vont s'installer sur un terrain inhospitalier qu'ils transformeront, par leur travail, en un petit paradis.

IV. pp. 339-356. Sir Bridway, noble britannique a un fils de vingt ans et une fille de seize. Son fils Guillaume prend parti pour la révolte du Duc de Monmouth. Celui-ci est défait par les troupes royales. L'épisode est suivi par une féroce et cruelle répression orchestrée par Kirke et Jeffreys. Guillaume est arrêté et condamné à mort. Les biens de son père sont confisqués. Hélène, la sœur de Guillaume essaie d'intercéder en leur faveur auprès de Kirke. Celui-ci abuse de la jeune fille après un mariage fictif. Guillaume est exécuté, sa mère meurt de chagrin, sa sœur devient folle et disparaît peu après. Accompagné seulement par son domestique Souval, Bridway est abandonné sur un tas d'ordures. À partir de ce moment, il gagnera sa vie comme cordonnier.

V. pp. 548-560. Cette nouvelle parisienne narre les aventures de la jeune Adelaïde. Cette fille d'un riche roturier est courtisée par le marquis de Lorvál, beau parleur et aventurier sans scrupules. Cependant, celui-ci, grâce à son talent et sa supposée noblesse est bien accueilli par le père de la jeune fille, flatté dans ses prétentions nobiliaires. Grâce à une ruse, il soustrait la jeune fille à ses parents imprudents puis il abuse d'elle. Croyant que ses parents la rejette, celle-ci doit ensuite se prostituer. Elle 
contracte la siphyllis et finit à l'hôpital de Bicêtre où elle rencontre Eusebio et Hardyl. Les deux héros l'aideront à revenir chez ses parents, qui sont prêts à lui accorder leur pardon. Hardyl servira d'intermédiaire avec la famille et dissimulera une partie de la vérité (la prostitution).

VI. pp. 675-85 : Lord Towsend était gouverneur du port du Plymouth, une charge obtenue grâce à la protection d'un haut personnage, lord M... Lorsque celui-ci meurt, il est remplacé par lord W... qui a servi sous les ordres de Towsend lorsqu'il était lieutenant de vaisseau. Il garde contre son ancien supérieur une rancune tenace, suite à un châtiment pourtant mérité et il va donc le faire accuser de haute trahison. Towsend apprend sa condamnation au moment où sa femme est sur le point de mourir. Son fidèle domestique Tautel organise la fuite de la famille en France. Ruinée, elle se retrouve dans le besoin à Paris et sera aidée par Eusebio et quelques nobles anglais.

\section{NOTES}

1. Vincent Jouve, "Voix et valeurs ", Nouvelles approches de la voix narrative, Narratologie, $\mathrm{n}^{\circ} 5$, Paris, L'Harmattan, 2003, p. 80.

2. Edmond Cros, La sociocritique, Paris, L'Harmattan, 2003, p. 53.

3. De ce point de vue, l'œuvre de référence est celle de Jorge Serra, Sur l'éducation philosophique dans l'Eusebio de Montengón, Besançon, Annales littéraires de l'Université de Besançon, 1991

4. "Nouvelles intercalées et récits secondaires dans le roman espagnol du 18ème siècle ", Les langues Néo-latines, n³18, 2001, pp. 39-57.

5. Jean Michel Adam, Le texte narratif, Paris, Nathan Université, 1994 ainsi que Les textes, types et prototypes, Paris, Nathan Université, 1997.

6. Marc Marti, « Nouvelles intercalées et récits secondaires dans le roman espagnol du 18ème siècle ", Les langues Néo-latines, n³18, 2001, pp.39-57. Afin de ne pas surcharger notre travail, nous laisserons de côté les problèmes de terminologie qui se posent en castillan et qui ont déjà été traités par d'autres auteurs. Voir à ce propos le travail de Jean Michel Laspéras, La nouvelle espagnole au siècle d'or, Montpellier, Publications de la recherche, 1987.

7. Voir Philippe Andrès, La nouvelle, Paris, Ellipses, 1996, pp. 4-6.

8. Dans le Don Quichotte, Cervantès propose, dans le troisième chapitre de la seconde partie, une brève réflexion sur la nouvelle « $\mathrm{El}$ curioso impertinente » qu'il a interpolée dans la première partie de son roman.

9. Jean-Louis Brau, Fonction des nouvelles intercalées dans le roman espagnol au siècle d'or, Nice, CNA, 1991, Cahiers de narratologie, n4. Voir aussi les deux travaux plus récents de Javier González Rovira «Poética y retórica del relato interpolado" Actas del IV Congreso Internacional de la Asociación Internacional Siglo de Oro (AISO) : (Alcalá de Henares, 22-27 de julio de 1996), Vol. 1, pp. 741-750 ainsi que celui de Juan Ramón Muñoz Sánchez « Los episodios de Los trabajos de Persiles y Segismunda », Hesperia: Anuario de filología hispánica, № 6, 2003, pp. 147-173.

10. Selon la classification définie par Gérard Genette, les narrateurs sont dans ce cas intrahétéro-diégétiques. Voir le tableau dans Figures III, Paris, Seuil, 1972, p. 256.

11. Jean-Louis Brau, Op. cit., p. 199.

12. Gérard Genette, Figures II, Paris, Seuil, 1969, p. 202.

13. Voir notre travail, art. cit., p. 40 sq.

14. Voir à ce propos les remarques au début de l'article de Ana L. Baquero Escudero, «Tres historias intercaladas y tres puntos de vista distintos en el primer Quijote ", Actas del segundo coloquio internacional de cervantistas, Álcalá de Henares 6-9 nov. 1989, Barcelona, Anthropos, 1991, pp. 417-423. 
15. Edmond Cros, Op. cit, p. 159.

16. Jean-Michel Adam, Les textes..., p. $56 \mathrm{sq}$

17. J.M. Adam, Ibid., p. 184.

18. J.M. Adam, Ibid., p. 185.

19. Voir dans ce même numéro de la revue le rappel que fait à ce propos A. Rabatel.

20. Vladimir Propp, Morphologie du conte, Paris, Seuil, [1965], 1970, p. 38.

21. Jean-Michel Adam, Les textes, ..., p. 51.

22. Dans l'article précédemment cité, Marc Marti, «Nouvelles intercalées... » nous avions délimité sept nouvelles. Après un nouvel examen, la nouvelle de l'aveugle (el tío Antón) peut être exclue car elle se déroule entièrement dans la diégèse. Il s'agit en fait d'un épisode secondaire, comparable au vol de la voiture en Angleterre.

23. Eusebio apprendra cependant à la fin du roman que la nouvelle d'Isidoro rancontée par Hardyl était en grande partie autobiographique.

24. La seule exception est celle de Luis Robert qui commet un larcin aux dépends d'Henrique Myden, père adoptif du héros, raconté p. 210.

25. Début de l'épisode, à la page 155 et fin racontée par J. Bridge p. 416.

26. Début de l'épisode p. 276.

27. Début de l'épisode, p. 370, fin p. 491.

28. L épisode est raconté de façon continue, pp. 645-659

29. Sur cet épisode parisien et ses significations, voir notre travail «París, ciudad etapa de un viaje educativo. Las imágenes de la capital francesa en Eusebio (1788) de Pedro Montengón», 28ème congrès de la SHF, Paris, mars 1997, París y el mundo ibérico e iberoamericano, Paris, U. de Paris X, 1998, pp. 349-358

30. Eusebio,p. 246.

31. Joaquín Álvarez Barrientos, in Francisco Aguilar Piñal (dir.), Historia de la literatura española, «La narrativa del siglo XVIII », Madrid Espasa Calpe, 1995, p. 955.

32. Voir Marc Marti, Nouvelles intercalées et récits secondaires dans le roman espagnol du 18 ème siècle ", art. cit.

33. On remarque cependant que ce personnage populaire prend en charge un certain nombre de récits de la trame principale : il raconte sa vie au début du roman tout comme il racontera, sur un ton tout aussi picaresque, le vol de la voiture en Angleterre.

34. Joaquín Álvarez Barrientos, La novela del siglo XVIII, Madrid, Júcar, 1992, p. 394.

35. Jorge Serra, Op. cit., p. 14 : « Aussi doit-on ajouter que l'admiration ressentie par l'auteur pour la méthode empiriste, le rend moins friand de longues constructions rhétoriques et plus enclin à l'utilisation de l'exemple qu'il considère, non sans raison, plus efficace, plus pratique pour montrer au lecteur le bien fondé des idées exposées dans le livre et pour conquérir de la sorte son adhésion ».

36. Horace, Ars Poetica, vv 343-344: "Omne tulit punctum qui miscuit utile dulci,/ Lectorem delectando, pariterque monendo ".

37. Dans un précédent travail, nous avions souligné les parallèles qu'on pouvait établir entre l'épisode parisien et le roman libertin français. Voir Marc Marti, « París, ciudad etapa », art. cit., pp. 357-358.

38. Les pratiques systématiques de ces deux techniques dans La Leandra, au début du $19^{\text {ème }}$ siècle ont été étudiées par Jerónimo Herrera, " "La Leandra“, novela de don Antonio Valladares de Sotomayor », Homenaje a Luis Morales Olivier, Madrid, FUE, 1986, pp. 623-641. 


\section{RÉSUMÉS}

Ce travail propose une analyse des relations entre les structures de la logique narrative et l'éthique dans un roman d'éducation espagnol du XVIIIe siiècle, Eusebio, de Pedro Montengón.

El artículo propone un análisis de las relaciones que se establecen entre las estructuras de la lógica narrativa y la ética en una novela educativa española del siglo 18, Eusebio, de Pedro Montengón.

\section{INDEX}

\section{Index géographique : Espagne}

Mots-clés : éthique, Eusebio, Littérature des Lumières, logique narrative, Montengón, nouvelles intercalées, roman éducatif

Index chronologique : XVIIIe siècle

\section{AUTEUR}

\section{MARC MARTI}

Centre de narratologie appliquée (EA 317), Université de Nice ; Principaux travaux sur l'Espagne des Lumières (Littérature et civilisation) 